Marquette University

e-Publications@Marquette

Biomedical Engineering Faculty Research and

Publications

Engineering, College of

$1-1-2014$

\title{
Age-related differentiation of sensorimotor control strategies during pursuit and compensatory tracking
}

Megan L. Heenan

Marquette University

Robert A. Scheidt

Marquette University, robert.scheidt@marquette.edu

Scott A. Beardsley

Marquette University, scott.beardsley@marquette.edu

Accepted version. Published as part of the proceedings of the conference, 201436 th Annual International Conference of the IEEE Engineering in Medicine and Biology Society (EMBC), 2014: 3562-3565. DOI. (C) 2014 Institute of Electrical and Electronics Engineers (IEEE). Used with permission. 
NOT THE PUBLISHED VERSION; this is the author's final, peer-reviewed manuscript. The published version may be accessed by following the link in the citation at the bottom of the page.

\title{
Age-related Differentiation Of Sensorimotor Control Strategies During Pursuit And Compensatory Tracking
}

\author{
Megan Heenan \\ Biomedical Engineering Department, Marquette University, Milwaukee, WI \\ Robert A. Scheidt \\ Biomedical Engineering Department, Marquette University, Milwaukee, WI \\ Scott A. Beardsley \\ Biomedical Engineering Department, Marquette University, Milwaukee, WI
}

\begin{abstract}
Motor control deficits during aging have been well-documented. Various causes of neuromotor decline, including both peripheral and central neurological deficits, have been hypothesized. Here, we use a model of closed-loop sensorimotor control to examine the functional causes of motor control deficits during aging. We recruited 14 subjects aged 19-61 years old to participate in a study in which they performed single-joint compensatory and pursuit tracking tasks with their dominant hand. We found that visual response delay and visual noise increased with age, while reliance on visual feedback, especially during compensatory tracking decreased. Increases in visual noise were also positively correlated with increases in movement error during a reach and hold task. The results suggest an increase in noise within the visuomotor control system may contribute to the decline in motor performance during early aging.
\end{abstract}


NOT THE PUBLISHED VERSION; this is the author's final, peer-reviewed manuscript. The published version may be accessed by following the link in the citation at the bottom of the page.

\section{SECTION I.}

\section{Introduction}

Age-related decline in performance during sensorimotor tasks is a well-known phenomenon. Older subjects tend to move more slowly, less accurately, with increased variability, ${ }^{1}$ and have difficulty coordinating complex movements. Older subjects also exhibit increased intermittency ${ }^{2}$ and increased reliance on visual feedback. ${ }^{3}$ Various hypotheses have been proposed to explain why older subjects exhibit motor changes; these include reduction in muscle force, ${ }^{4}$ increased muscle noise, ${ }^{5}$ deteriorated sensory feedback, cognitive slowing, reduced white ${ }^{6,7}$ and grey ${ }^{7,8}$ matter, and altered movement goals. ${ }^{5}$

Here we examine movement performance during two types of task: compensatory tracking, in which subjects correct for an applied perturbation, and pursuit tracking, in which subjects follow a moving target. Previous research has shown that these two tasks use different modes of control.9,10 Pursuit and compensatory tracking exhibit specialization, with the left hemisphere (in right-handed subjects) specializing in feedforward movement planning, while the right hemisphere monitors online control for corrections to movement error. ${ }^{10}$ Examination of movement parameters during both types of tasks therefore provides valuable insight into the control of movement during aging.

\section{SECTION II.}

\section{Methods}

\section{A. Model Description}

Sensorimotor control of I-D movement was modeled as a multi - input, single-output linear system, which has previously been shown to approximate continuous, single-joint movement. ${ }^{11,12}$ The model (Fig. 1) consisted of a feed-forward control path, two sensory feedback pathways, and a forward model to compensate for sensory delays. Inputs to the system consisted of desired position $\left(\theta_{\mathrm{d}}\right)$ and an external perturbation $\left(\mathrm{D}_{\text {ext }}\right)$. Feedback of actual limb position $\left(\theta_{\mathrm{a}}\right)$ was summed with the external perturbation before being delayed $\left(\mathrm{T}_{\mathrm{v}}, \mathrm{T}_{\mathrm{p}}\right)$ and weighted $\left(\mathrm{K}_{\mathrm{v}}, \mathrm{K}_{\mathrm{p}}\right)$. Uncertainty in sensory estimates of position was characterized by separate visual (white) and proprioceptive (Pink) noise sources. Delays in sensory feedback were compensated for using a forward model to generate a delayed prediction of limb position. An inverse model (PID controller) generated corrective torques 
in response to instantaneous estimates of position error. A $2^{\text {nd }}$ order model of dynamics about the elbow was used to map the applied torques to changes in arm position.

\section{B. Subjects}

Fourteen healthy volunteers (10 female) aged 19-61 years (mean $37.6 \pm 16.9$ years) participated in compensatory and pursuit tracking tasks. Thirteen subjects were right handed, according to the Edinburgh handedness inventory; one was ambidextrous. Written informed consent was obtained from each subject in accordance with institutional guidelines approved by the Marquette University Institutional Review Board.

\section{Setup}

Subjects performed a series of compensatory and pursuit tracking tasks about the right elbow joint using a I-D robotic manipulandum. The goal of all tasks was to stabilize a computer generated cursor on a visual target. During pursuit tracking, a perturbation (0$10 \mathrm{~Hz}$ band-limited white noise) was applied to the target position. During compensatory tracking, the cursor, rather than the target, was perturbed. In both cases, subjects were asked to bring the cursor to the target as quickly and accurately as possible.

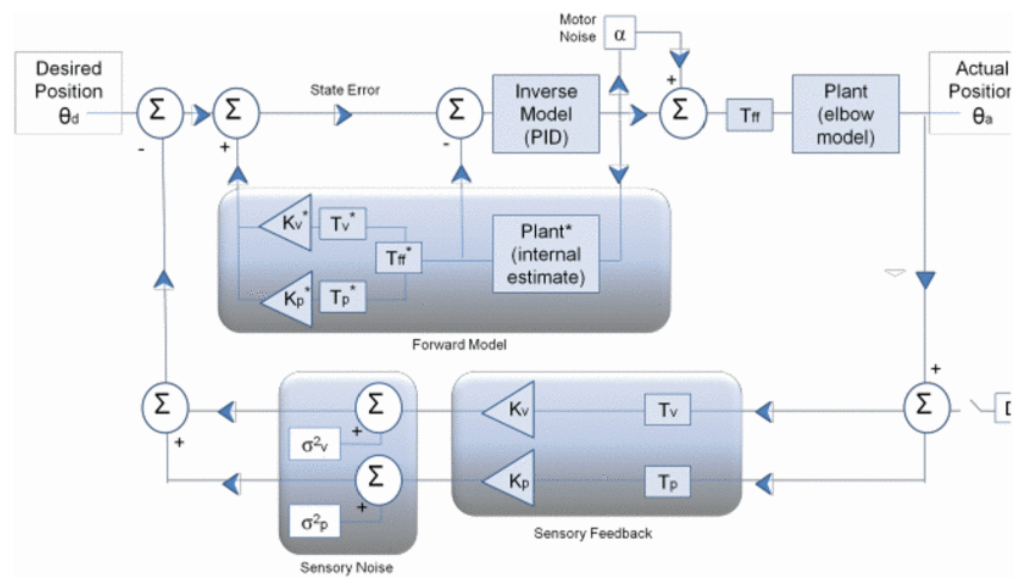

Figure 1. Multi-input, single output model of 1-d sensorimotor control of movement. The model is characterized by fourteen free parameters including weighted and delayed sensory feedback, system noise, and a compensatory forward model.

\section{Task Description}

Subjects participated in one, two-hour session consisting of four experiments designed to characterize the model parameters. For the purposes of analysis, subjects were 
grouped into five age groups: $18-25(N=4), 26-35(N=3), 36-45(N=$ 2),46-55 $(N=1)$, and 56- $65(N=4)$.

\section{1) Feedforward Motor Noise}

Subjects performed 25 eight-second isometric elbow flexion trials, during which they moved the cursor (under torque control) to capture a stationary displaced target. Five trials were obtained at each of five different torque levels $(2,4,6,8$, and $10 \mathrm{~N}-\mathrm{m})$. The average within-trial standard deviation of each torque level was calculated from the last 5 seconds of torque data, during which the subjects' position and torque was constant. The gain of the feedforward motor noise was calculated as the slope of the linear least-squares fit of standard deviation vs. torque level.

\section{2) Sensory Response Delays}

In the second experiment, three separate tasks were used to estimate sensory response delays. A continuous, low frequency ( $0-1 \mathrm{~Hz}$ white noise), pseudorandom perturbation was applied to either the cursor (visual compensation), target (visual pursuit), or manipulandum (proprioceptive compensation) position. Ten 20-second trials were collected for each condition. Sensory response delays were estimated from the visual and proprioceptive compensatory tasks by finding the offset of the peak cross-correlation between the perturbation and subjects'responses. 
NOT THE PUBLISHED VERSION; this is the author's final, peer-reviewed manuscript. The published version may be accessed by following the link in the citation at the bottom of the page.

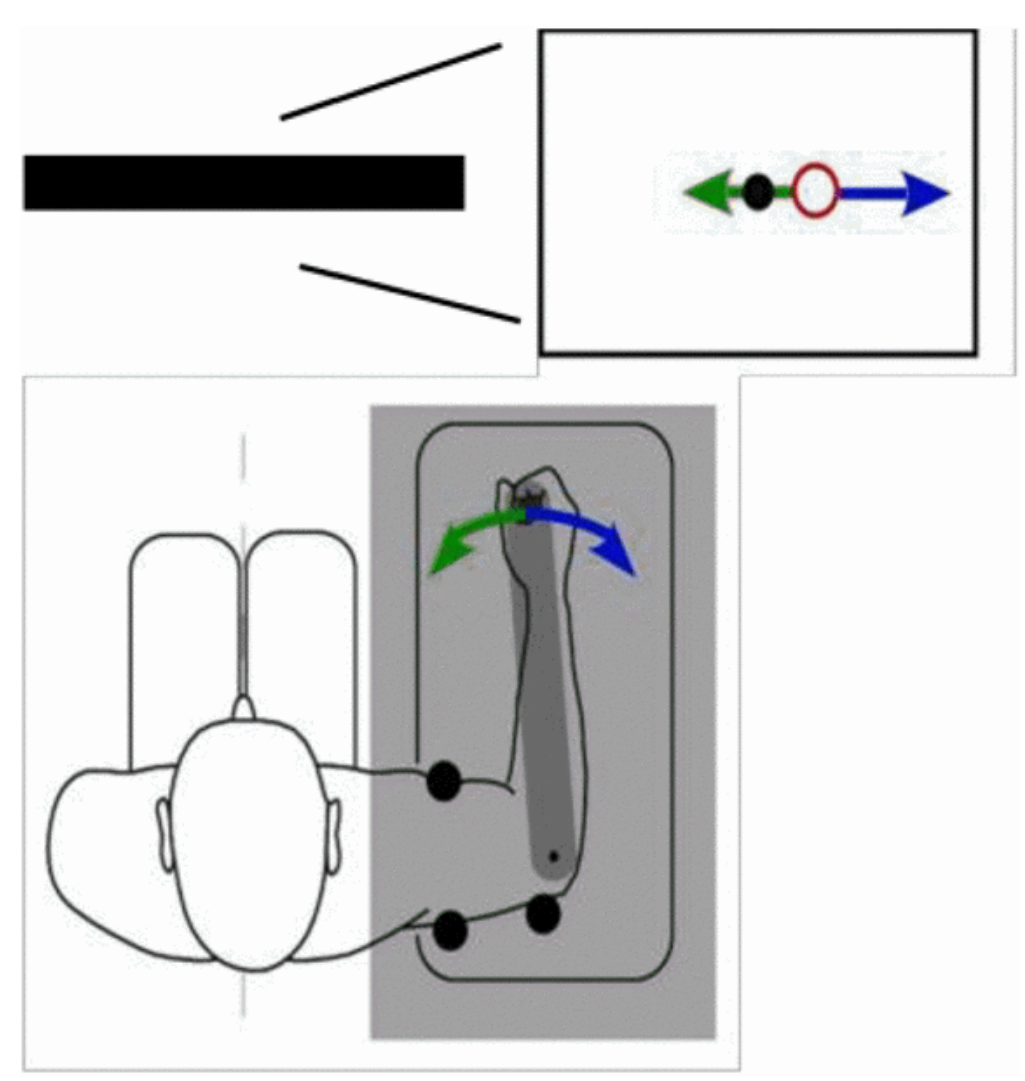

Figure 2. Experimental setup. Subjects were seated facing a computer monitor and held a 1D manipulandum with their right hand. During the tasks subjects used rotations about their elbow to bring a cursor (red ring) to a target (black dot) as continuous perturbations were applied to the system.

\section{3) Active Elbow Dynamics, Sensory Gains, Neural Controller Gains, and Sensory Noise}

The third experiment consisted of two tasks (one pursuit, one compensatory), each of which contained ten, 32 -second trials. A high frequency $(0-10 \mathrm{~Hz}$, filtered at $1 \mathrm{~Hz}$ with a $2^{\text {nd }}$ order zero-phase Butterworth filter) pseudorandom perturbation was applied to either the cursor or target position. Subjects were asked to bring the cursor to the target as quickly and accurately as possible. For each set of trials, three separate frequency response functions (FRF s) were calculated. Each FRF was calculated from the data on a trial-by-trial basis, then all individual FRFs were averaged across trials. The first FRF related each subjects' final position to their input torque and was used to characterize the passive dynamics of the elbow joint. A $2^{\text {nd }}$-order model of wrist dynamics

$$
\text { Plant }=\frac{1}{J s^{2}+B s+K}
$$

(Eq. 1)

2014 Annual International Conference of the IEEE Engineering in Medicine and Biology Society (EMBC), (2014): 3562-3565. DOI. This article is (C) Institute of Electrical and Electronics Engineers (IEEE) and permission has been granted for this version to appear in e-Publications@Marquette. Institute of Electrical and Electronics Engineers (IEEE) does not grant permission for this article to be further copied/distributed or hosted elsewhere without the express permission from Institute of Electrical and Electronics Engineers (IEEE). 
where model parameters J, B, and K correspond to the moment of inertia, viscous damping, and spring constant of the elbow joint, respectively, was fit to the FRF using the simplex method.

A bootstrapping analysis was used to characterize the uncertainty in the leastsquares fit and compare parameter estimates between subjects. During the bootstrapping analysis, data were resampled with replacement ten thousand times. Each resampled data set was fit using randomized initial conditions, to obtain a distribution of estimates for each parameter. The mean and standard deviation of the resulting distributions were calculated for subsequent analysis.

The second FRF characterized the model transfer function relating the applied perturbation (Dext for compensatory movements and $\theta_{d}$ for pursuit movements) and subject's arm position, for stabilization (Eq. 2) and pursuit (Eq. 3) respectively. The FRFs were fit using the same method described above.

$$
\begin{array}{r}
\theta_{a}=\frac{K_{v} e^{-T_{v} s} C s D_{\text {ext }}}{\frac{s}{P}+C s}(\text { Eq. } 2) \\
\theta_{a}=\frac{e^{-T_{v} s} C s \theta_{d}}{\frac{s}{P}+C s}(\text { Eq.3) }
\end{array}
$$

where $\mathrm{P}$ is the transfer function of the plant (Eq. 1) and $\mathrm{C}$ is the transfer function of the PID controller (derivative gain: $\mathrm{K}_{\mathrm{d}}$; proportional gain: $\mathrm{K}_{P r}$; integral gain. $\mathrm{K}_{\mathrm{i}}$ )

$$
C=K_{d} s+K_{p r}+\frac{K_{i}}{s}
$$

We performed a simultaneous fit of the final FRF, which related the noise at the output of the system to the desired position, to estimate $D_{v}$ (the variance of additive white noise) and $\mathrm{D}_{\mathrm{p}}$ (the variance of additive $\mathrm{l} / \mathrm{f}$ noise), constraining $\mathrm{K}_{\mathrm{v}}, \mathrm{K}_{\mathrm{p}} \mathrm{K}_{\mathrm{d}}, \mathrm{K}_{\mathrm{pr}}$ " and $\mathrm{K}_{\mathrm{i}}$ to be equal in both fits. 


\section{4) Movement Error}

The final experiment characterized subjects' movement speed and steady-state error during a reach-and-hold task. Subjects performed ten, 10-second trials during which the target was randomly displaced to the left or the right of the subjects' midline over a range of \pm 40 degrees. Subjects were instructed to center the cursor on the target as quickly and accurately as possible.

For each target displacement, steady-state movement error was calculated as the mean squared error of the movement after the subject had moved the hand to within 1.5 degrees of the desired target. Error was then averaged across all target displacements and trials.

\section{SECTION III.}

\section{Results}

\section{A. Feedforward Motor Noise}

Feedforward motor noise did not increase significantly with age group $\left(\left(\mathrm{r}^{2}=\right.\right.$ $0.16 ; \mathrm{p}=0.51$ ). The gain of the motor noise across subjects was $0.035 \pm 0.028$ for the youngest age group and $0.027 \pm 0.011$ for the oldest age group.

\section{B. Sensory Response Delays}

There was a trend toward increased visual response delay with age group (Fig. 3, left) that fell just above the threshold for significance $(r=0.52 ; \mathrm{P}=0.068)$. However, proprioceptive response delay showed no consistent variation with age $(\mathrm{r}=0.047 ; \mathrm{p}=$ 0.88), (Fig. 3, right).

\section{Active Elbow Dynamics, Sensory Gains, Neural Controller Gains, Sensory Noise}

Average FRFs for compensatory and pursuit tasks differed across age groups. In younger subjects (subjects $<45$ years), FRFs for compensatory and pursuit tasks were nearly identical (Fig. 4, left). In the oldest subjects (subjects $>55$ years), the compensatory response was attenuated compared to the pursuit response, while the pursuit response was similar to that of the younger subjects (Fig. 4, right). Part of the attenuation in FRF 
during compensatory tracking of the visual perturbation was due to a significant decrease in visual gain among subjects aged $>55\left(\mathrm{t}(12)=5.13 ; \mathrm{P}<0 \_001\right)$.
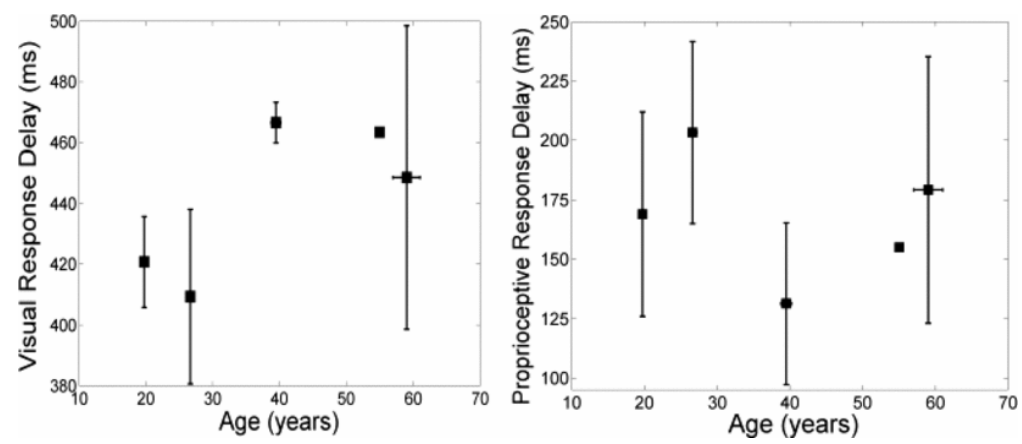

Figure 3. Sensory response delay vs.age group. (L) Visual response delay. (R) Proprioceptive response delay. Error bars denote standard deviation across subjects.
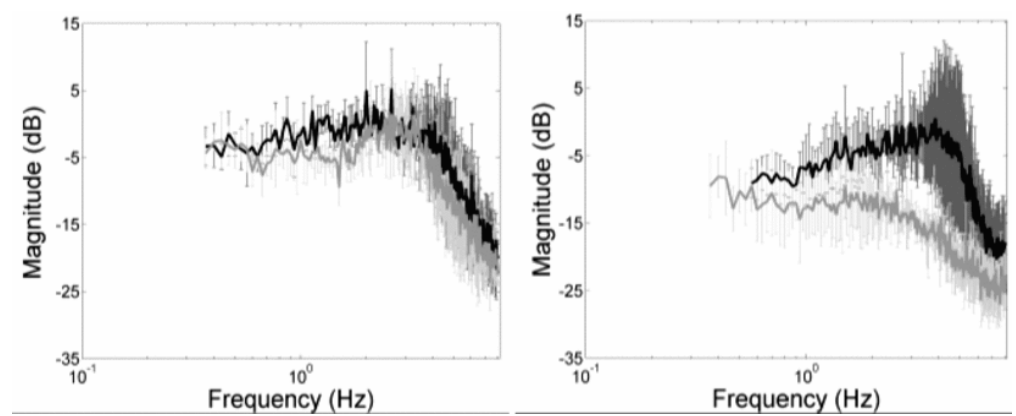

Figure 4. Average FRFacross younger (left; $<45$ years) and older (right; $>55$ years) subjects for pursuit (black) and compensatory (grey) tracking. Error bars denote standard deviation across subjects.

During compensatory tracking, derivative, proportional, and integral gains of the neural controller increased in subjects older than 55 (Fig. 5). During pursuit tracking, controller gains remain constant across age groups. The change in controller parameters with age during compensatory, but not pursuit, tasks, suggests that older subjects exhibit more task differentiation than younger subjects. We also found that the variance in the visual noise increased significantly with age group $(r=0.79 ; p=0.0038)$ (Fig. 6, left), while the variance in proprioceptive noise did not $(r=-0.37 ; p=0.21)$.

\section{Movement Error}

Mean endpoint error (compensatory error) during the reach and hold task increased significantly with age $(r=0.61 ; \mathrm{p}=.028)$. Endpoint error was positively correlated with age-related changes in visual $(r=0.65 ; \mathrm{p}=0.030)$ (Fig. 7 , right), but not proprioceptive noise $(r=0.44 ; p=0.13)$. 


\section{SECTION IV.}

\section{Discussion}

Here, we used systems identification techniques in the context of a 14-parameter sensorimotor control model to examine the effects of aging on pursuit and compensatory tracking. We found significant changes in how older subjects process visual feedback. Specifically, visual processing delays increased with age, visual processing noise increased with age and the weight placed on visual feedback decreased with age. Interestingly, visual gain was similar for subject groups $<55$, but exhibited a sharp decrease for subject groups $>55$. This may indicate an aging-related breakdown in the ability of the brain to compensate for increased processing noise and larger delay times.

As visual feedback gain decreased, controller gains increased to achieve comparable levels of error reduction to that of younger subjects. The increases in controller gains during compensatory tracking could reflect deliberate changes in strategy on the part of older subjects, who tend to move more slowly and with a greater focus on accuracy. In particular, an increase in integral gain in the neural controller may indicate a greater focus on endpoint error (which integral gain is meant to minimize) at the expense of movement speed (derivative gain).
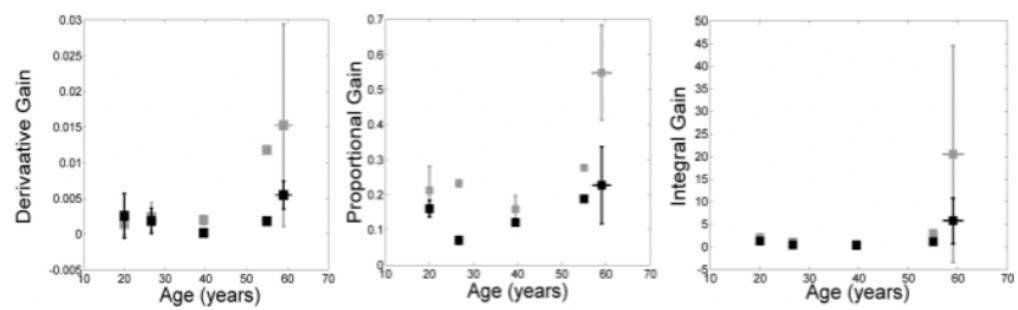

Figure 5. Controller gains vs. Age group. Derivative (left), proportional (middle), and integral (right) gains during pursuit (black) and compensatory (grey) tasks. Error bars denote standard deviation.
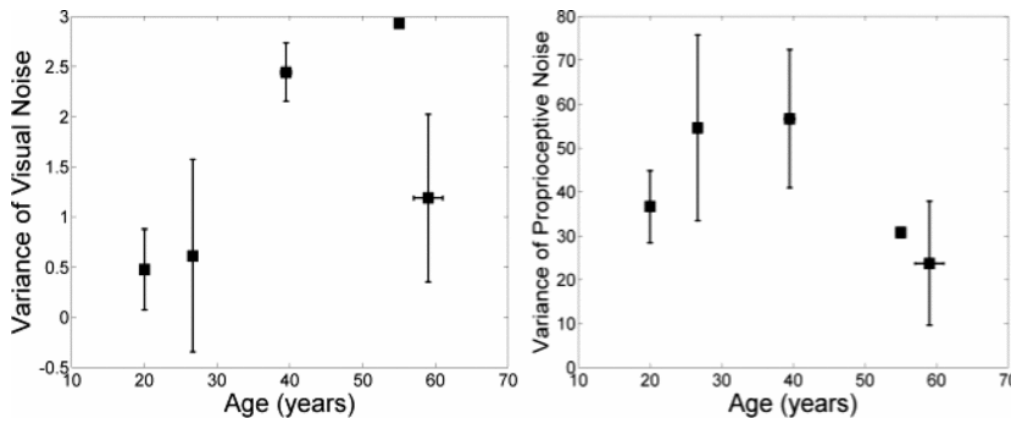

2014 Annual International Conference of the IEEE Engineering in Medicine and Biology Society (EMBC), (2014): $3562-3565$. DOI. This article is (C) Institute of Electrical and Electronics Engineers (IEEE) and permission has been granted for this version to appear in e-Publications@Marquette. Institute of Electrical and Electronics Engineers (IEEE) does not grant permission for this article to be further copied/distributed or hosted elsewhere without the express permission from Institute of Electrical and Electronics Engineers (IEEE). 
Figure 6. Variance of sensory noise. Internal visual (left) and proprioceptive (right) noise varience vs. Age group. Error bars denote standard deviation.
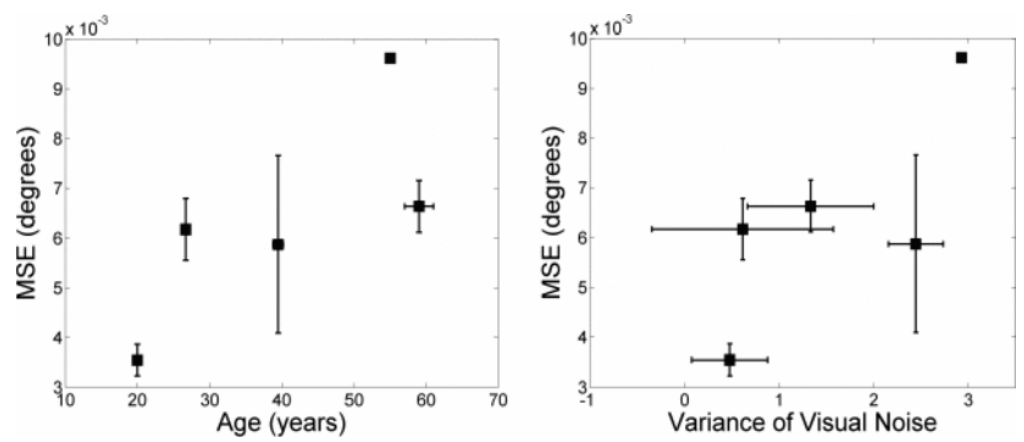

Figure 7. Movement error. Steady-state error vs. Age (left) and variance of the subjects' internal visual noise (right). Error bars denote standard deviation.

By contrast, we found no correlation between age and motor noise, proprioceptive delay times, or proprioceptive noise. These results suggest that changes in the peripheral nervous system are unlikely to be the cause of age-related changes in movement performance.

Our results suggest that changes in visual feedback systems may underlie altered task performance during early aging. This is supported by the fact that older subjects exhibit the greatest changes in performance during compensatory and not pursuit tracking. Pursuit tracking may rely more on open-loop control, minimizing the effects of noisy or inaccurate visual feedback.

\section{SECTION V.}

\section{Conclusion}

Using systems identification with a sensorimotor control model of goal-directed movement, we have shown that older subjects exhibit differences in neuromotor control (relative to younger subjects) during compensatory - but not pursuit - tracking. Additionally, early signs of aging in movement control may include degradation of the visuomotor processing system, resulting in slower movement times, greater movement error, and alterations in neural control strategy (e.g. altered controller gains). 


\section{References}

${ }^{1}$ Caroline J. Ketcham, Rachel D. Seidler, Arend W.A. Van Gemmert, George E. Stelmach, "Age-related kinematic differences as influenced by task difficulty target size and movement amplitude", $L$ Geron, vol. 57B, pp. 54-64, 2002.

2Matthieu P. Boisgontier, Vincent Nougier, "Ageing of internal models: from a continuous to an intermittent proprioceptive control of movement", Age (Dordr), vol. 35, pp. 1339-55, Aug. 2013.

${ }^{3}$ Rachel O. Coats, P. Warm, "The reliance on visual feedback control by older adults is highlighted in tasks requiring precise endpoint placement and precision grip", Exp. Brain Res., vol. 214, pp. 139-50, 2011.

${ }^{4}$ Lee Gyusung, Fradet Laetitia, J. Ketcham Caroline, Dounskaia Natalia, "Efficient control of arm movements in advanced age", Exp. Brain Res., vol. 177, pp. 78-94, 2007.

5Walker Neff, A. Philbin David, D. Fisk Arthur, "Age-related differences n movement control: adjusting submovement structure to optimize performance", J. Geron, vol. 52B, pp. 40-52, 1997.

6Bartzokis George, H. Lu Po, Tingus Kathleen, F. Mendez Mario, Richar Auore, G. Peters Douglas, Oluwadara Bolanle, A. Barrall Katherine, J. Paul Finn, Villablanca Pablo, M. Thompson Paul, Mintz Jim, "Lifespan trajectory of myelin integrity and maximum motor speed", Neurobiology of Aging., vol. 31, pp. 1554-62, 2010.

7Jill Campbell Stewart, Xuan Tran, Steven C. Cramer, "Age-related variability in performance of a motor action selection task is related to differences in brain function and structure among older adults", Neuroimage, vol. 86, pp. 326-334, 2014.

8 F. Hoffstaedter, C. Grefkes, C. Roski, S. Caspers, K. Zilles, S.B. Eickhoff, "Age-related decrease of functional connectivity additional to gray matter atrophy in a network for movement initiation", Brain Struct. Funct., 2014.

${ }^{9}$ Robert A. Scheidt, Claude Ghez, "Separate Adaptive Mechanisms for Controlling Trajectory and Final Position in Reaching", J. Neurophysiol., vol. 98, pp. 3600-3613, 2007.

10Sydney Y. Schaefer, Y. Haaland Kathleen, L. Sainburg Robert, "Dissociation of Initial Trajectory and Final Position Errors during Visuomotor Adaptation Following Unilateral Stroke", Brain Research, pp. 78-91, 2009.

11R.J. Peterka, "Sensorimotor Integration in Human Postural Control", J. Neurophysiol., vol. 88, pp. 1097118, 2002.

12M.L. Heenan, R.A. Scheidt, S.A. Beardsley, "Visual and proprioceptive contributions to compensatory and pursuit tracking movements in humans", Conf. Proc IEEE Eng. Med. Biol. Soc., 2011. 